bioRxiv preprint doi: https://doi.org/10.1101/531848; this version posted January 28,2019 . The copyright holder for this preprint (which was not certified by peer review) is the author/funder, who has granted bioRxiv a license to display the preprint in perpetuity. It is made available under aCC-BY-NC-ND 4.0 International license.

\title{
1 Inhibiting IFT dynein with ciliobrevin in C. elegans chemosensory cilia
}

2 Jona Mijalkovic and Erwin J.G. Peterman*

3 LaserLaB and Department of Physics and Astronomy, Vrije Universiteit Amsterdam, De

4 Boelelaan 1081, $1081 \mathrm{HV}$, Amsterdam, The Netherlands

$5 \quad{ }^{*}$ Corresponding author: e.j.g.peterman@vu.nl

6

7

8

9

10

11

12

13

14

15

16

17

18

19 


\section{Abstract}

21 Cytoplasmic dyneins play a role in a myriad of cellular processes, such as retrograde intracellular transport and cell division. Small-molecule cytoplasmic dynein antagonists, ciliobrevins, have recently been developed as tools to acutely probe cytoplasmic dynein function. Although widely used to investigate cytoplasmic dynein 1, far fewer studies explore the effect of ciliobrevin on cytoplasmic dynein 2 or IFT dynein. Here, we use ciliobrevin A to partially disrupt IFT dynein in the chemosensory cilia of living C. elegans. Acute, lowconcentration ciliobrevin treatment results in shortening of cilia and reduction of transport velocity in both directions. After longer exposure to ciliobrevin, we find concentrationdependent motor accumulations and axonemal deformations. We propose that maintenance of ciliary length requires a high fraction of active IFT-dynein motors, while structural integrity can be preserved by only a few active motors.

\section{Introduction}

Cytoplasmic dyneins are large ( 1.4MDa), multi-subunit, microtubule minus-end directed ATP-driven motor proteins with a wide range of vital, cellular functions [1]. Cytoplasmic dynein 1 plays, for example, a role in the axonal transport of cargo (e.g. membranous organelles, mRNA and proteins) and cellular positioning of the mitotic spindle, chromosomes and centrosomes. A second type of cytoplasmic dynein, called cytoplasmic dynein 2 or IFT dynein, drives retrograde intraflagellar transport (IFT) in cilia and flagella [2], cellular structures that act as antennae to detect and respond to changes in the extracellular environment and require motor-driven IFT for their assembly and maintenance [3-5].

Recent advances in understanding cytoplasmic dynein function in vivo have largely relied on gene modification tools such as MosSCI, CRISPR and expression regulation using RNAi [6- 
9]. An important limitation of these methods is that they induce long-term cellular changes and thus cannot be used to probe the effect of acute and controlled (partial) dynein loss of function. To overcome this, small-molecule cytoplasmic dynein antagonists have been developed, enabling real-time motor inhibition [10-12]. Ciliobrevins are dihydroquinazolinone compounds discovered in a high-throughput screen for Hedgehog-signaling $(\mathrm{Hh})$ pathway inhibitors [13]. In this screen, mouse fibroblast Hh-responsive cells treated with HPI-4 (later renamed ciliobrevin A) displayed shorter or absent cilia and accumulated transcription factor Gli2 and IFT-B particle subunit IFT88, pointing to perturbed retrograde transport [10, 13]. Ciliobrevin $A$ and its derivative ciliobrevin $D$ have since been utilized to investigate dynein-1driven processes in cultured cells, such as chromosome segregation [14, 15], axonal transport and elongation [16] and centrosome orientation [17, 18]. Ciliobrevin was additionally found to inhibit IFT-mediated gliding motility and membrane-protein transport in Chlamydomonas, highlighting its potential as a dynein-2 inhibitor in vivo $[19,20]$. However, the use of ciliobrevins in other living organisms to probe IFT-dynein function has remained underexplored.

Here, we test the efficacy of ciliobrevin A as IFT-dynein inhibitor in living C. elegans and examine the effect of acute dynein perturbation on IFT-motor ensemble distributions and velocities. We find that IFT in $C$. elegans phasmid chemosensory cilia is not halted by acute, low-concentration ciliobrevin treatment, but slows down, resulting in shortening of the ciliary axoneme. After prolonged ciliobrevin exposure, we find concentration-dependent motor accumulations and axonemal deformations indicative of more severely impaired transport. Based on these findings, we propose that maintenance of maximum ciliary length requires a high fraction of active IFT-dynein motors, while structural integrity can be preserved by only a few active motors. 


\section{Results}

\section{Optimization of ciliobrevin A dosing in C. elegans}

72 First, we sought to determine the dose required to exert an inhibitory effect on IFT dynein in

C. elegans phasmid cilia. Nematodes expressing fluorescently-tagged IFT-dynein light intermediate chain XBX-1 (XBX1::GFP) were initially treated with 20-100 $\mu \mathrm{M}$ ciliobrevin $\mathrm{A}$, the concentration range reported to give maximum inhibitory response in different cell lines $[10,13,16]$. The animals were incubated in a ciliobrevin-containing solution or ciliobrevincoated Nematode Growth Medium (NGM) plates with OP50 E. coli for 1 hour and then imaged using epifluorescence microscopy [21, 22]. We subsequently screened for changes in phasmid cilium length using long-exposure fluorescence images of XBX-1::EGFP. Visual inspection of the fluorescence images revealed that, in contrast to the cell lines studied before, no ciliary shortening was observed at these concentrations in $C$. elegans, suggesting that ciliobrevin does not elicit an inhibitory effect in this concentration range in the nematode. Exposure to increasingly higher doses resulted in observable ciliary shortening at concentrations above $0.7 \mathrm{mM}, \sim 3-7$ times higher than typically used in cell lines $[10,13,16]$. This higher concentration could suggest that ciliobrevin has a lower affinity for $C$. elegans IFT dynein, but it more likely reflects limited penetration of the drug or absorption-related drug loss in the nematode. Moreover, it is consistent with dosing regimens of other small-molecule drugs in C. elegans that have been reported to be in the millimolar-range [23, 24]. 


\section{Acute ciliobrevin treatment results in roadblocks, ciliary 93 shortening and impaired bidirectional transport}

94 To examine the effect of minimal but acute IFT-dynein inhibition, nematodes were subjected

95

96

97 to 5-minute treatment with a "low", $0.7 \mathrm{mM}$ ciliobrevin dose (Fig 1A). Ciliobrevin is soluble in DMSO, a solvent that is toxic to $C$. elegans at concentrations above $2 \% \mathrm{~V} / \mathrm{V}$ [25]. To test whether effects were specific to ciliobrevin and not induced by the $1 \%$ V/V DMSO used in the ciliobrevin experiments, we also treated nematodes with a control solution containing the same amount of DMSO. After dosing, the nematodes were allowed to recover briefly on unseeded NGM plates (2-3 minutes) and then anesthetized for 10 minutes using levamisole (Fig 1A). Ciliobrevin-induced ciliary shortening was quantified using long-exposure fluorescence images of XBX-1::EGFP (Fig 1B). In untreated nematodes, XBX1::EGFP is localized specifically to the chemosensory cilia and is distributed across the entire cilium [21, 22]. In control-treated nematodes, the average length occupied by XBX-1 was $8.33 \pm 0.34$ $\mu \mathrm{m}$ (average \pm s.e.m), in agreement with previous length determination of $C$. elegans cilia $[22,26]$. After acute, low-concentration ciliobrevin A treatment, the average length occupied markedly reduced to $5.85 \pm 0.44 \mu \mathrm{m}$ (Fig $1 \mathrm{C}$ ). Our results confirm that, similar to observations in Chlamydomonas and ciliated cells, ciliobrevin can induce ciliary shortening in C. elegans [19]. 


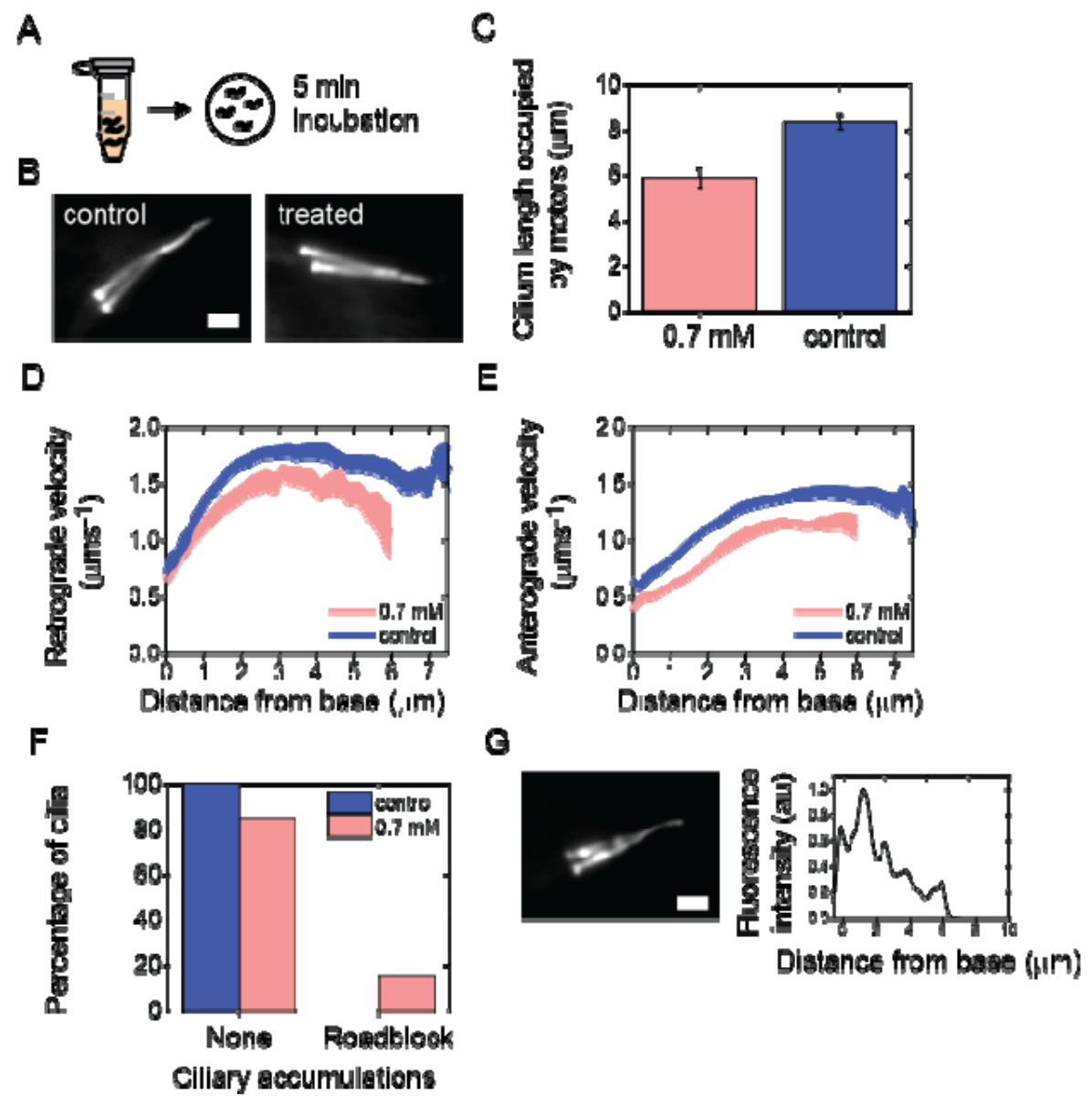

110

111 Fig 1. Acute ciliobrevin treatment results in roadblocks and ciliary shortening.

112 (A) Dosing schematic: 5-minute exposure to $0.7 \mathrm{mM}$ ciliobrevin A or control (2\% DMSO in M9). (B) Example of summed fluorescence intensity images (150 subsequent images) of XBX-1 (XBX-1::EGFP) in cilia of treated and control animals. Scale bar $2 \mu \mathrm{m}$. (C) Average length occupied by XBX-1 after exposure to $70 \mathrm{mM}$ ciliobrevin $\mathrm{A}$ (light red; $\mathrm{n}=11$ cilia) or control (blue; $n=4$ cilia). Error is s.e.m. (D) Extent of $X B X-1$ accumulation in worms treated with $70 \mathrm{mM}$ ciliobrevin A (light red) and control (2\% (V/V) DMSO in M9; blue). (D) Average retrograde XBX-1 velocity after exposure to $70 \mathrm{mM}$ ciliobrevin $A$ ( $n=38$ trains from 11 cilia; 8 worms) and control ( $\mathrm{n}=24$ trains from 4 cilia; 4 worms).

(E) Average anterograde $\mathrm{XBX}-1$ velocity after exposure to $70 \mathrm{mM}$ ciliobrevin $A$ ( $n=44$ trains from 11 cilia; 8 worms) and control ( $n=24$ trains from 4 cilia; 4 worms). Line thickness represents s.e.m. (F) Example 
122

123

124

125

summed fluorescence intensity image of XBX-1 (XBX-1::EGFP) and corresponding normalized fluorescence intensity profile showing roadblock accumulations. Scale bar $2 \mu \mathrm{m}$.

To determine whether the velocity of IFT trains is affected by acute ciliobrevin treatment, we next generated direction-filtered kymographs and extracted position-dependent velocities of XBX-1::EGFP using KymographClear and KymographDirect (Fig 1D, E) [27]. In the retrograde direction (tip to base, Fig 1D) IFT dynein is the active driver of transport, but in the anterograde direction (base to tip, Fig 1E) IFT dynein is carried as cargo by the kinesin-2 family motors OSM-3 and kinesin-II. We found that retrograde IFT-train velocity is reduced by $\sim 12 \%$, suggesting rapid inhibition of (at least some) IFT-dynein motors participating in transport. Interestingly, kinesin-2-driven anterograde transport was also reduced ( 21\%). While the original report by Firestone et al suggested that ciliobrevin $A$ and $D$ do not affect kinesin-1-dependent microtubule gliding in in vitro assays with only kinesin-1, our data is consistent with studies in primary neurons showing that ciliobrevin D affects both anterograde and retrograde transport [16, 28]. Similarly, in Chlamydomonas, anterograde transport impairment occurs already 2 minutes after treatment [19]. It is possible that ciliobrevin is not specific to the cytoplasmic dynein ATP binding site, but interpretation of these findings is difficult owing to the strong interdependence between anterograde and retrograde transport [29]. Functional IFT requires continuous turnover and impairment of retrograde transport could thus indirectly lead to impairment of anterograde transport [30]. Another possibility is that anterograde IFT trains require functional, uninhibited dynein motors [31].

Next, we used the long-exposure fluorescence images to visualize the effect of ciliobrevin A on XBX-1 distribution. In all control-treated cilia there were no visible accumulations of motors along the cilium (Fig 1B, F). In 15.4\% of the acutely ciliobrevin-treated cilia, however, we observed XBX-1 accumulations at non-specific locations along the cilium (Fig 1F, G). We attribute these "roadblock" accumulations to inhibition of a small subset of IFT-dynein motors, 
resulting in stalled IFT trains. These non-moving IFT trains can be bypassed by active motors (Movie S1), indicating that they do not completely block IFT. Taken together, our findings affirm the efficacy of ciliobrevin $A$ in the phasmid cilia of living $C$. elegans. Importantly, we show that acute IFT-dynein inhibition using a minimal ciliobrevin concentration does not completely halt IFT. While the number of functional dynein motors is likely diminished, IFT is maintained, albeit at a lower velocity, resulting in a reduced ciliary length.

\section{Prolonged ciliobrevin A treatment leads to concentration-}

\section{and velocity-dependent ciliary shortening}

To explore the effect of more prolonged inhibition of IFT dynein, we subjected nematodes to 1 hour treatment with low- and high-concentration ciliobrevin (0.7 and $1.4 \mathrm{mM}$ in $2 \%$ DMSO, respectively), or $2 \%$ DMSO solution as control. Due to the limited solubility of ciliobrevin and DMSO toxicity (above $2 \% \mathrm{~V} / \mathrm{V}$ ), $1.4 \mathrm{mM}$ was the highest ciliobrevin concentration attainable in our experiments. In control-treated nematodes, the average distance occupied by motors was $8.25 \pm 0.18 \mu \mathrm{m}$, similar to control experiments with $1 \%$ DMSO (Fig 2A). After 1-hour treatment with $0.7 \mathrm{mM}$ and $1.4 \mathrm{mM}$ ciliobrevin, cilia appeared shorter and the average length occupied by XBX-1::EGFP reduced to $6.91 \pm 0.17 \mu \mathrm{m}$ and $6.11 \pm 0.30 \mu \mathrm{m}$ respectively. To probe whether the observed shortening is due to motor retraction or axonemal shortening, we also exposed animals with labeled ciliary tubulin TBB-4::EGFP to $1.4 \mathrm{mM}$ ciliobrevin solution. The average cilium length in these animals was $5.59 \pm 0.22 \mu \mathrm{m}$ (Fig 2A), confirming that ciliobrevin induces retraction of the axoneme. To obtain further insight into ciliary shortening, we plotted the distribution of post-treatment length occupied by XBX-1 for each treatment (Fig 2A). While in all low-concentration-treated worms XBX-1 extended from base 
174 to beyond the proximal segment, in some high-concentration treated worms we observed

$175 \mathrm{XBX}-1$ only occupying the first $2-4 \mu \mathrm{m}$ of the cilium, most likely indicating retraction of (part

176 of) the proximal segment. Such severe truncations were not observed with acute or

177 prolonged low-concentration treatment, revealing concentration-dependent ciliary shortening

178 in response to prolonged ciliobrevin treatment (Fig 1C, Fig 2A).

179

180

181

182

183

184

185

186

187

188 
A<smiles></smiles>

60 min incubation

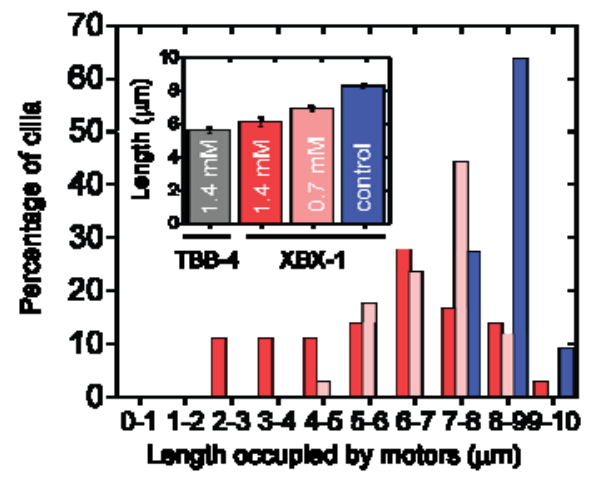

B

C
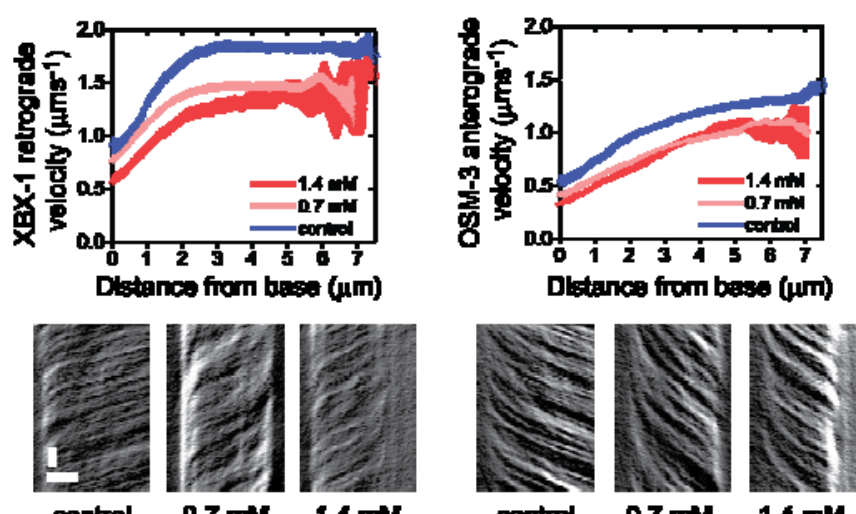

D

E
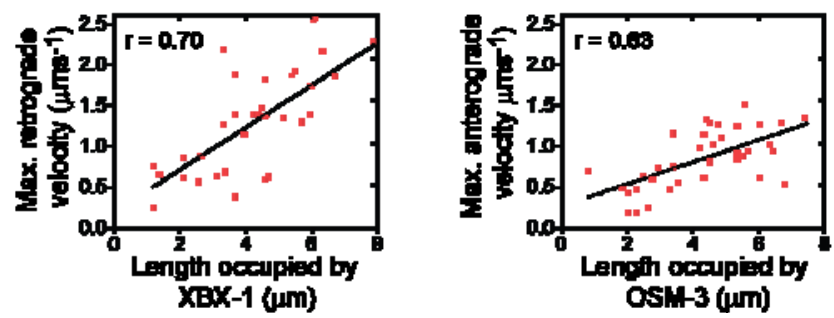

Fig 2. Concentration- and velocity-dependent ciliary shortening after ciliobrevin A exposure.

(A) Dosing schematic: 60-minute exposure to ciliobrevin A or control (2\% DMSO in M9). Inset: Average cilium length (TBB-4, grey, $n=39$ ) and average ciliary length occupied by XBX-1 after 60-minute exposure to $0.7 \mathrm{mM}$ ciliobrevin A (light red, $\mathrm{n}=34$ ), $1.4 \mathrm{mM}$ ciliobrevin (red, $n=39$ ) or control (blue). Error is s.e.m. Graph: Distribution of cilium lengths occupied by XBX-1 after 60-minute exposure to ciliobrevin A or control. (B) Train-averaged XBX-1 retrograde velocity after exposure to $0.7 \mathrm{mM}$ ciliobrevin $A$ ( $n=130$ trains from 34 cilia; 18 
198

199

200

201

202

203

204

205

206

207

208

209

210

211

212

213

214

215

216

217

218

219

220

221

222

223

worms), $1.4 \mathrm{mM}$ ciliobrevin A ( $\mathrm{n}=138$ trains from 35 cilia; 19 worms) and control $(\mathrm{n}=79$ trains from 11 cilia; 7 worms). Line thickness represents s.e.m. Representative corresponding kymographs are shown. Time: vertical; scale bar $2 \mathrm{~s}$. Position: horizontal; scale bar $2 \mu \mathrm{m}$. (C) Train-averaged OSM-3 anterograde velocity and representative kymographs after exposure to $0.7 \mathrm{mM}$ ciliobrevin A ( $\mathrm{n}=136$ trains from 34 cilia; 18 worms), $1.4 \mathrm{mM}$ ciliobrevin $A$ ( $n=146$ trains from 35 cilia; 19 worms) and control ( $n=44$ trains from 11 cilia; 7 worms). Line thickness represents s.e.m. (D-E) Maximum XBX-1 (D) and OSM-3 (E) velocity versus ciliary length occupied by motors. Red squares: data points; black lines represent regression lines obtained from a Pearson's correlation analysis (correlation coefficient $r$ : 0.70 for XBX-1 and 0.63 for OSM-3).

We next investigated the effect of prolonged ciliobrevin treatment on IFT-motor velocity using a dual-label C. elegans strain with fluorescently-tagged IFT dynein and one of the anterograde kinesin-2 motors, OSM-3 [22]. We recorded fluorescence image sequences of IFT dynein (XBX-1::EGFP) and OSM-3 (OSM-3::mCherry) and generated kymographs. Position-dependent velocities were then extracted using KymographClear and KymographDirect (Fig 2B, C) [27]. Prolonged treatment with ciliobrevin resulted in a 24\% (at $0.7 \mathrm{mM}$ ) and $\sim 36 \%$ (at $1.4 \mathrm{mM}$ ) lower retrograde IFT-dynein velocity (Fig 2B). The anterograde (OSM-3) velocity was also reduced, by $\sim 31 \%$ (at $0.7 \mathrm{mM}$ ) and $\sim 34 \%$ (at $1.4 \mathrm{mM}$;

Fig 2C). These results show that higher concentrations of ciliobrevin and longer exposure to the drug result in a larger reduction of the velocity in both directions. To further explore the relationship between cilium length and IFT velocity, we plotted the maximum anterograde (Fig 2D) and retrograde (Fig 2E) velocity for each cilium. We observe a clear trend (Pearson's $r=0.70$ and 0.63 for XBX-1 and OSM-3 respectively) between maximally attained IFT velocities and length of cilium occupied by motors, suggesting that the extent of ciliary shortening could be velocity-dependent. 


\section{Prolonged ciliobrevin A exposure leads to altered motor}

\section{5 distribution}

226 To investigate the effect of prolonged ciliobrevin A exposure on IFT-dynein distribution, we

227 characterized the extent of IFT-dynein accumulations after high- and low-concentration

228 treatment (Fig 3). As expected, in control-treated nematodes there were no aberrant motor

229 accumulations (2\% DMSO; Fig 3A). In nematodes that underwent prolonged, low-

230 concentration treatment, $50 \%$ of phasmid cilia had no motor accumulations and $29 \%$ had

231 "roadblock" accumulations at apparently random positions along the cilium. In a smaller

232 fraction of the cilia (9\%) we observed IFT-dynein accumulations only at the ciliary tip (Fig

233 3B), consistent with defective retrograde transport. The observation of tip accumulation of

234 IFT components upon ciliobrevin treatment is consistent with earlier studies in cell lines[10]

235 and Chlamydomonas [19]. In some cilia (12\%), motor accumulation in the distal segment

236 was more substantial, covering at least $1 \mu \mathrm{m}$ of the distal segment including tip (Fig 3C). In

237 nematodes that underwent prolonged, high-concentration ciliobrevin treatment we observed

238 more severe disruptions of the IFT-dynein distribution. 13\% of the cilia showed motor

239 accumulation only at the tip, while in $33 \%$ of cilia dynein motors had stalled in the entire distal

240 segment, substantially affecting the integrity of the axoneme (Fig 3C). We hypothesize that in

241 these nematodes there are too few active IFT-dynein motors left to maintain bidirectional IFT,

242 resulting in (partial) collapse of the axoneme. In some cases, we observed a remarkable

243 heterogeneity in the response to ciliobrevin between the two cilia in a phasmid pair within

244 one organism, despite identical exposure. In the example shown in Fig 3B, one phasmid

245 cilium is substantially shortened while the other has maintained its full length. These

246 observations suggest that there is a balance point in the number of functional IFT

247 components required for distal segment maintenance and structural integrity (Fig $3 F$ ). In this

248 view, only a small fluctuation of component numbers below this balance point, in a given

249 cilium at a given time, will result in collapse of IFT and ciliary structure. In addition, after 
250 prolonged high-concentration treatment (but not in acute or prolonged low-concentration

251 treatment) we observed structural aberrations of the cilia in some nematodes, such as tight

252 bends (Fig 3E). This could be due to a ciliobrevin-induced effect on tubulin structure or

253 disruption of IFT-mediated tubulin transport [16, 32] at these high concentrations.

A

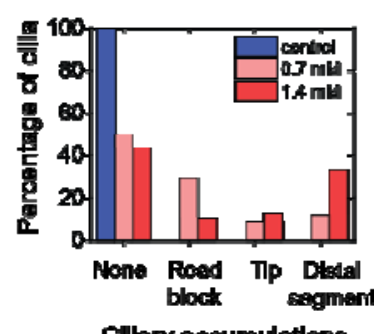

C

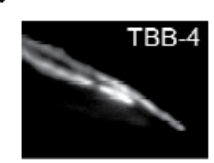

D

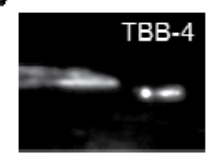

E
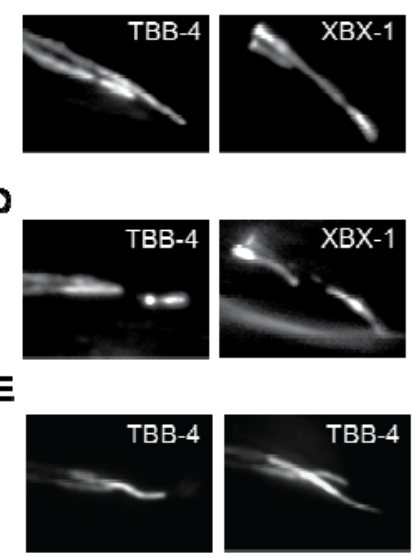

$\mathbf{F}$
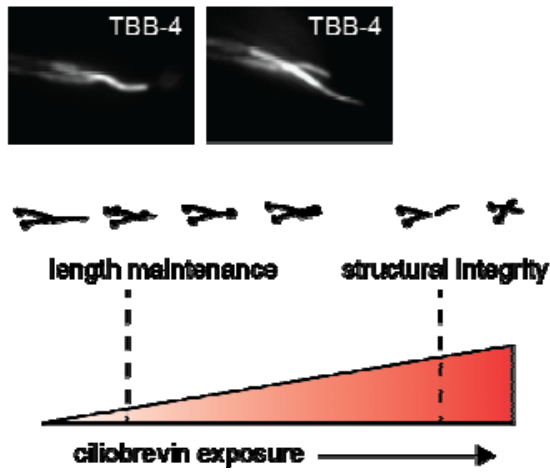

BB-4

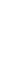

B
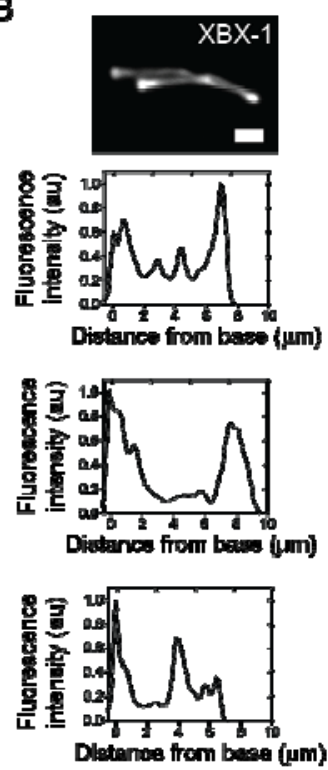

254 
261 of TBB-4 after $1.4 \mathrm{mM}$ ciliobrevin treatment showing axonemal malformations. Scale bar 2

$262 \mu \mathrm{m}$. (F) Model of ciliobrevin-induced IFT-dynein inhibition on IFT. The axonemal length

263 maintenance balance-point is easily disturbed, whereas structural integrity can be maintained

264 with a much lower number of active IFT-dynein motors.

Taken together, our findings enable us to propose a model of the ciliary effects of IFT-dynein inhibition by ciliobrevin. Acute, low-concentration treatment results in axonemal shortening coupled with a reduction in IFT velocity in both directions, suggesting that both maximum ciliary length and IFT-velocity maintenance require a relatively high number of active IFTdynein motors. At higher ciliobrevin concentrations, motors become slower and cilia become even shorter, until only a few active motors remain, tipping the balance of ciliary structure, resulting in severely truncated cilia with axonemal defects.

\section{Discussion}

275 The recent development of ciliobrevins has made it possible to acutely inhibit cytoplasmic 276 dynein in several cellular systems [10]. Here, we show that ciliobrevin A, dosed in the low millimolar range, can exert an inhibitory effect on IFT dynein in C. elegans phasmid chemosensory cilia.

Although we have shown that ciliobrevin A can inhibit IFT dynein in living $C$. elegans, there are several caveats to using it as a quantitative and well-controllable tool to probe dynein function. Firstly, the pharmacokinetics of ciliobrevin in $C$. elegans is unknown and ciliobrevin might not be specific to one cytoplasmic dynein subtype. Therefore, if uptake primarily takes place through the mouth instead of the ciliary openings, dynein-1-driven function and dendritic transport could also be affected. Additionally, the absolute intracellular ciliobrevin concentration is unknown and could be up to 40 times lower than the extracellular dose [24]. 
286 Secondly, in IFT, motors work in teams of several tens of motors and we cannot currently

287 determine the number of motors actively engaged in transport. As such, ciliobrevin

288 concentration is the only surrogate for the number of (in)active motors, but this relationship

289 may not necessarily be linear.

290 Notwithstanding these limitations, we have made some clear observations of the effects of 291 ciliobrevin on IFT in C. elegans phasmid cilia. The first effects of acute ciliobrevin treatment are that IFT velocity has decreased $12-21 \%$, both in anterograde and retrograde direction, and that cilia are $\sim 30 \%$ shorter. These effects occur before IFT motors accumulate at the ciliary tip, as we observe after longer exposure, consistent with previous studies on cells [10].

As a consequence, these first effects represent the initial response of the IFT system to minimal IFT-dynein perturbation. A lower IFT-dynein velocity can be attributed to frictional drag or less efficient collective motion due to ciliobrevin-induced inhibition of a subset of motors [33]. The lower velocity appears to correlate with the retraction of the axoneme. This suggests that a minimum IFT-train velocity (and potentially a minimum number of active IFTdynein motors) is required for the maintenance of the full length of the cilium. Reduced IFT velocities have also been observed in Chlamydomonas mutants with shorter flagella[34]. In Chlamydomonas experiments where flagellar shortening was induced by a $\mathrm{pH}$ shock, long flagella also had higher IFT velocities than shorter flagella [35]. IFT trains, however, were larger in short than in long flagella, leading to a balance-point model based on train size [35]. To connect our findings with those in Chlamydomonas, it would be interesting to detect how many motors are actively engaged with the axoneme at a given time on an IFT train, but such tools are currently lacking in vivo.

Our results suggest that IFT is relatively robust. Despite inhibition of a subset of IFT-dynein motors, bidirectional transport still continues, albeit at a lower velocity, resulting in a shorter axoneme. Such adaptations to mild perturbations of ciliary processes could be necessary for quick responses to extracellular changes. 


\section{Materials and Methods}

\section{C. elegans strains and dosing}

C. elegans were maintained according to standard procedures. Nematodes were grown at $20^{\circ} \mathrm{C}$ on Nematode Growth Medium (NGM) plates seeded with Escherichia coli OP50 bacteria. The strains used in this study, EJP212 (XBX-1::EGFP, OSM-3::mCherry) [22] and EJP401 (TBB4::EGFP), were generated using Mos1-mediated single-copy insertion (MosSCI) [36]. Ciliobrevin A powder (H4541, Sigma Aldrich) was dissolved in fresh DMSO at maximum solubility to make a $140 \mathrm{mM}$ stock solution stored at $-20^{\circ} \mathrm{C}$ for a maximum of 1 month (after 1 month we observed loss of efficacy). The ciliobrevin dosing solution was made by diluting this stock solution in $\mathrm{M} 9$ to $1.4 \mathrm{mM}(2 \%$ (V/V) DMSO content) or $0.7 \mathrm{mM}(1 \%$ DMSO content) and used the same day. Control (vehicle) dosing solutions were made by dissolving DMSO in M9 (1\% and 2\% DMSO). Young adult hermaphrodites were dosed by transferring to standard $0.2 \mathrm{ml}$ PCR tubes (Thermo Scientific) with $0.7 \mathrm{mM}$ or $1.4 \mathrm{mM}$ ciliobrevin solution for $5 \mathrm{~min}$ or $60 \mathrm{~min}$. Nematodes were allowed to recover on unseeded NGM plates for 2-3 minutes post-dosing.

\section{Fluorescence imaging and analysis} in M9 pad covered with a $22 \times 22 \square \mathrm{mm}$ cover glass and sealed with VaLaP. Fluorescence imaging was done using a custom-built epi-illuminated fluorescence microscope as described previously $[22,26]$. Fluorescence images were analyzed using KymographDirect and KymographClear [27]. 


\section{Acknowledgements}

We acknowledge financial support from the Netherlands Organization for Scientific Research (NOW) via a Vici grant (E.J.G.P.). We thank Pierre Mangeol for help with the dosing set-up in the initial stages of the project.

\section{References}

1. Cianfrocco MA, DeSantis ME, Leschziner AE, Reck-Peterson SL. Mechanism and Regulation of Cytoplasmic Dynein. Annual review of cell and developmental biology. 2015;31:83-108. Epub 2015/10/06. doi: 10.1146/annurev-cellbio-100814-125438. PubMed PMID: 26436706; PubMed Central PMCID: PMCPmc4644480.

2. Hou Y, Witman GB. Dynein and intraflagellar transport. Experimental cell research. 2015;334(1):26-34. Epub 2015/03/01. doi: 10.1016/j.yexcr.2015.02.017. PubMed PMID: 25725253; PubMed Central PMCID: PMCPmc4433774.

3. Singla V, Reiter JF. The primary cilium as the cell's antenna: signaling at a sensory organelle. Science (New York, NY). 2006;313(5787):629-33. Epub 2006/08/05. doi: 10.1126/science.1124534. PubMed PMID: 16888132.

4. Scholey JM. Intraflagellar transport. Annual review of cell and developmental biology. 2003;19:423-43. Epub 2003/10/23. doi: 10.1146/annurev.cellbio.19.111401.091318. PubMed PMID: 14570576.

5. Rosenbaum JL, Witman GB. Intraflagellar transport. Nature reviews Molecular cell biology. 2002;3(11):813-25. Epub 2002/11/05. doi: 10.1038/nrm952. PubMed PMID: 12415299.

6. He Y, Francis F, Myers KA, Yu W, Black MM, Baas PW. Role of cytoplasmic dynein in the axonal transport of microtubules and neurofilaments. The Journal of cell biology. 2005;168(5):697-703. Epub 2005/02/25. doi: 10.1083/jcb.200407191. PubMed PMID: 15728192; PubMed Central PMCID: PMCPmc2171826.

7. $\mathrm{Li} \mathrm{W}, \mathrm{Yi} \mathrm{P}, \mathrm{Ou} \mathrm{G}$. Somatic CRISPR-Cas9-induced mutations reveal roles of embryonically essential dynein chains in Caenorhabditis elegans cilia. The Journal of cell biology. 2015;208(6):683-92. Epub 2015/03/18. doi: 10.1083/jcb.201411041. PubMed PMID: 25778918; PubMed Central PMCID: PMCPmc4362450.

8. Hao L, Efimenko E, Swoboda P, Scholey JM. The retrograde IFT machinery of C. elegans cilia: two IFT dynein complexes? PloS one. 2011;6(6):e20995. Epub 2011/06/23. doi: 10.1371/journal.pone.0020995. PubMed PMID: 21695221; PubMed Central PMCID: PMCPmc3112216.

9. Reck J, Schauer AM, VanderWaal Mills K, Bower R, Tritschler D, Perrone CA, et al. The role of the dynein light intermediate chain in retrograde IFT and flagellar function in Chlamydomonas. Molecular biology of the cell. 2016;27(15):2404-22. Epub 2016/06/03. doi: 10.1091/mbc.E16-03-0191. PubMed PMID: 27251063; PubMed Central PMCID: PMCPmc4966982.

10. Firestone AJ, Weinger JS, Maldonado M, Barlan K, Langston LD, O'Donnell M, et al. Small-molecule inhibitors of the AAA+ ATPase motor cytoplasmic dynein. Nature. 2012;484(7392):125-9. Epub 2012/03/20. doi: 10.1038/nature10936. PubMed PMID: 22425997; PubMed Central PMCID: PMCPmc3321072.

11. See SK, Hoogendoorn S, Chung AH, Ye F, Steinman JB, Sakata-Kato T, et al. Cytoplasmic Dynein Antagonists with Improved Potency and Isoform Selectivity. ACS 
379 chemical biology. 2016;11(1):53-60. Epub 2015/11/12. doi: 10.1021/acschembio.5b00895.

PubMed PMID: 26555042; PubMed Central PMCID: PMCPmc4715766.

12. Hoing S, Yeh TY, Baumann M, Martinez NE, Habenberger P, Kremer L, et al. Dynarrestin, a Novel Inhibitor of Cytoplasmic Dynein. Cell chemical biology. 2018;25(4):35769.e6. Epub 2018/02/06. doi: 10.1016/j.chembiol.2017.12.014. PubMed PMID: 29396292.

13. Hyman JM, Firestone AJ, Heine VM, Zhao Y, Ocasio CA, Han K, et al. Smallmolecule inhibitors reveal multiple strategies for Hedgehog pathway blockade. Proceedings of the National Academy of Sciences of the United States of America. 2009;106(33):14132-7. Epub 2009/08/12. doi: 10.1073/pnas.0907134106. PubMed PMID: 19666565; PubMed Central PMCID: PMCPmc2721821.

14. Sikirzhytski V, Magidson V, Steinman JB, He J, Le Berre M, Tikhonenko I, et al. Direct kinetochore-spindle pole connections are not required for chromosome segregation. The Journal of cell biology. 2014;206(2):231-43. Epub 2014/07/16. doi: 10.1083/jcb.201401090. PubMed PMID: 25023516; PubMed Central PMCID: PMCPmc4107786.

15. Miao Y, Zhou C, Cui Z, Tang L, ShiYang X, Lu Y, et al. Dynein promotes porcine oocyte meiotic progression by maintaining cytoskeletal structures and cortical granule arrangement. Cell cycle (Georgetown, Tex). 2017;16(21):2139-45. Epub 2017/09/22. doi: 10.1080/15384101.2017.1380133. PubMed PMID: 28933593; PubMed Central PMCID: PMCPmc5731405.

16. Sainath R, Gallo G. The dynein inhibitor Ciliobrevin D inhibits the bidirectional transport of organelles along sensory axons and impairs NGF-mediated regulation of growth cones and axon branches. Developmental neurobiology. 2015;75(7):757-77. Epub 2014/11/19. doi: 10.1002/dneu.22246. PubMed PMID: 25404503; PubMed Central PMCID: PMCPmc4436090.

17. Yi J, Wu X, Chung AH, Chen JK, Kapoor TM, Hammer JA. Centrosome repositioning in T cells is biphasic and driven by microtubule end-on capture-shrinkage. The Journal of cell biology. 2013;202(5):779-92. Epub 2013/08/28. doi: 10.1083/jcb.201301004. PubMed PMID: 23979719; PubMed Central PMCID: PMCPmc3760611.

18. Liu X, Kapoor TM, Chen JK, Huse M. Diacylglycerol promotes centrosome polarization in T cells via reciprocal localization of dynein and myosin II. Proceedings of the National Academy of Sciences of the United States of America. 2013;110(29):11976-81. Epub 2013/07/03. doi: 10.1073/pnas.1306180110. PubMed PMID: 23818610; PubMed Central PMCID: PMCPmc3718145.

19. Shih SM, Engel BD, Kocabas F, Bilyard T, Gennerich A, Marshall WF, et al. Intraflagellar transport drives flagellar surface motility. eLife. 2013;2:e00744. Epub 2013/06/26. doi: 10.7554/eLife.00744. PubMed PMID: 23795295; PubMed Central PMCID: PMCPmc3679542.

20. Cao M, Ning J, Hernandez-Lara Cl, Belzile O, Wang Q, Dutcher SK, et al. Unidirectional ciliary membrane protein trafficking by a cytoplasmic retrograde IFT motor and ciliary ectosome shedding. eLife. 2015;4. Epub 2015/02/18. doi: 10.7554/eLife.05242. PubMed PMID: 25688564; PubMed Central PMCID: PMCPmc4362204.

21. Schafer JC, Haycraft CJ, Thomas JH, Yoder BK, Swoboda P. XBX-1 encodes a dynein light intermediate chain required for retrograde intraflagellar transport and cilia assembly in Caenorhabditis elegans. Molecular biology of the cell. 2003;14(5):2057-70. Epub 2003/06/13. doi: 10.1091/mbc.E02-10-0677. PubMed PMID: 12802075; PubMed Central PMCID: PMCPmc165097.

22. Mijalkovic J, Prevo B, Oswald F, Mangeol P, Peterman EJ. Ensemble and singlemolecule dynamics of IFT dynein in Caenorhabditis elegans cilia. Nature communications. 2017;8:14591. Epub 2017/02/24. doi: 10.1038/ncomms14591. PubMed PMID: 28230057.

23. Bridi JC, Barros AG, Sampaio LR, Ferreira JC, Antunes Soares FA, Romano-Silva MA. Lifespan Extension Induced by Caffeine in Caenorhabditis elegans is Partially Dependent on Adenosine Signaling. Frontiers in aging neuroscience. 2015;7:220. Epub 2015/12/24. doi: 10.3389/fnagi.2015.00220. PubMed PMID: 26696878; PubMed Central PMCID: PMCPmc4672644. 
433 24. Davies AG, Pierce-Shimomura JT, Kim H, VanHoven MK, Thiele TR, Bonci A, et al. A central role of the BK potassium channel in behavioral responses to ethanol in C. elegans. Cell. 2003;115(6):655-66. Epub 2003/12/17. PubMed PMID: 14675531.

25. Dengg M, van Meel JC. Caenorhabditis elegans as model system for rapid toxicity assessment of pharmaceutical compounds. Journal of pharmacological and toxicological methods. 2004;50(3):209-14. Epub 2004/11/03. doi: 10.1016/j.vascn.2004.04.002. PubMed PMID: 15519907.

26. Prevo B, Mangeol P, Oswald F, Scholey JM, Peterman EJ. Functional differentiation of cooperating kinesin-2 motors orchestrates cargo import and transport in C. elegans cilia. Nature cell biology. 2015;17(12):1536-45. Epub 2015/11/03. doi: 10.1038/ncb3263. PubMed PMID: 26523365.

27. Mangeol P, Prevo B, Peterman EJ. KymographClear and KymographDirect: two tools for the automated quantitative analysis of molecular and cellular dynamics using kymographs. Molecular biology of the cell. 2016. Epub 2016/04/22. doi: 10.1091/mbc.E1506-0404. PubMed PMID: 27099372.

28. Roossien DH, Lamoureux P, Miller KE. Cytoplasmic dynein pushes the cytoskeletal meshwork forward during axonal elongation. Journal of cell science. 2014;127(16):3593-602. doi: $10.1242 /$ jcs. 152611 .

29. Ocbina PJ, Eggenschwiler JT, Moskowitz I, Anderson KV. Complex interactions between genes controlling trafficking in primary cilia. Nature genetics. 2011;43(6):547-53. Epub 2011/05/10. doi: 10.1038/ng.832. PubMed PMID: 21552265; PubMed Central PMCID: PMCPmc3132150.

30. Marshall WF, Rosenbaum JL. Intraflagellar transport balances continuous turnover of outer doublet microtubules: implications for flagellar length control. The Journal of cell biology. 2001;155(3):405-14. Epub 2001/10/31. doi: 10.1083/jcb.200106141. PubMed PMID: 11684707; PubMed Central PMCID: PMCPmc2150833.

31. Ling SC, Fahrner PS, Greenough WT, Gelfand VI. Transport of Drosophila fragile X mental retardation protein-containing ribonucleoprotein granules by kinesin-1 and cytoplasmic dynein. Proceedings of the National Academy of Sciences of the United States of America. 2004;101(50):17428-33. Epub 2004/12/08. doi: 10.1073/pnas.0408114101. PubMed PMID: 15583137; PubMed Central PMCID: PMCPmc536039.

32. Hao L, Thein M, Brust-Mascher I, Civelekoglu-Scholey G, Lu Y, Acar S, et al. Intraflagellar transport delivers tubulin isotypes to sensory cilium middle and distal segments. Nature cell biology. 2011;13(7):790-8. Epub 2011/06/07. doi: 10.1038/ncb2268. PubMed PMID: 21642982; PubMed Central PMCID: PMCPmc3129367.

33. Mallik R, Petrov D, Lex SA, King SJ, Gross SP. Building complexity: an in vitro study of cytoplasmic dynein with in vivo implications. Current biology : CB. 2005;15(23):2075-85. Epub 2005/12/08. doi: 10.1016/j.cub.2005.10.039. PubMed PMID: 16332532.

34. Iomini C, Babaev-Khaimov V, Sassaroli M, Piperno G. Protein particles in Chlamydomonas flagella undergo a transport cycle consisting of four phases. The Journal of cell biology. 2001;153(1):13-24. Epub 2001/04/04. PubMed PMID: 11285270; PubMed Central PMCID: PMCPmc2185522.

35. Engel BD, Ludington WB, Marshall WF. Intraflagellar transport particle size scales inversely with flagellar length: revisiting the balance-point length control model. The Journal of cell biology. 2009;187(1):81-9. Epub 2009/10/07. doi: 10.1083/jcb.200812084. PubMed PMID: 19805630; PubMed Central PMCID: PMCPmc2762100.

36. Frokjaer-Jensen C, Davis MW, Hopkins CE, Newman BJ, Thummel JM, Olesen SP, et al. Single-copy insertion of transgenes in Caenorhabditis elegans. Nature genetics. 2008;40(11):1375-83. Epub 2008/10/28. doi: 10.1038/ng.248. PubMed PMID: 18953339; PubMed Central PMCID: PMCPmc2749959. 